\title{
sciendo
}

\author{
BULGARIAN ACADEMY OF SCIENCES
}

CYBERNETICS AND INFORMATION TECHNOLOGIES • Volume 20, No 1

Sofia $\bullet 2020$

Print ISSN: 1311-9702; Online ISSN: 1314-408

DOI: $10.2478 /$ cait-2020-00

\section{A Smart Social Insurance Big Data Analytics Framewor Based on Machine Learning Algorithms}

Youssef Senousy ${ }^{1}$, Abdulaziz Shehab ${ }^{2}$, Wael K. Hanno Alaa M lad ${ }^{1}$, Hazem A. El-bakry', Nashaat Elkhamisy²

${ }^{1}$ Department of Computers and Information Systems, University of $M_{1}$ nsoura, $M$ oura, Egypt

${ }^{2}$ Department of Information Systems, Sadat Academy for Management Sciences, Ca Egypt E-mails: youssef_senousy@hotmail.com abdulaziz_sheha mans.edu.eg wael_karam1@yahoo.com amriad2014@g al.com elbakrycrans.edu.eg wessasalsol@gmail.com

Abstract: Social insurance is an individua protection a inst risks such as retirement, death or disability. Big data mining d analytic tre a way that could help the insurers and the actua get the on fision for the insured individuals. Dependently, this pa $r$. Egyptian Social insurance big data VOSI' ... nntains data, which need some pre-processing methods after $e_{\lambda}$ ar on like replacing missing values, standardization and or er/e the da paper also presents using some mining methods, such as ofering d classi, ation algorithms on the Egyptian social insurance datase trough ar xneriment. clustering, we used K-means clustering and the result sho d a 0.138 with two clusters in the dataset features. In assifica. we used the Support Vector Machine (SVM) classifier and classific on results sho d a high accuracy percentage of $94 \%$.

Key rds: cial Insuranc Data Integration, Big Data Mining and Big Data Analytics.

\section{In duction}

cial insur ce is one of the branches of the insurance sciences; its programs rovide $\mathfrak{p}$ cetion against wage loss resulting from retirement, prolonged disability, a h. unemployment, and protection against the cost of medical care during old age disability [1]. The Social Insurance Authority in Egypt seeks to provide incured individuals, pensioners and their dependents with social protection as a replacement for revenue that is disrupted if one of the insured risks occurs to them. Fig. 1 illustrates the life cycle of the insured individual. The timeline of working periods is consisting of durations (job start date and job end date) and every duration has its salary value and salary date. The Age of working individuals ranges from 18 
to 60 for employees and 18 to 65 for employer owner. Pensions is the end of the individual life cycle. The good thing about the pension system is the income of the pensions is not given only to the pension owner but also his/her family as wife, sons, and parents, who we call, pension beneficiaries if the pension owner dies.

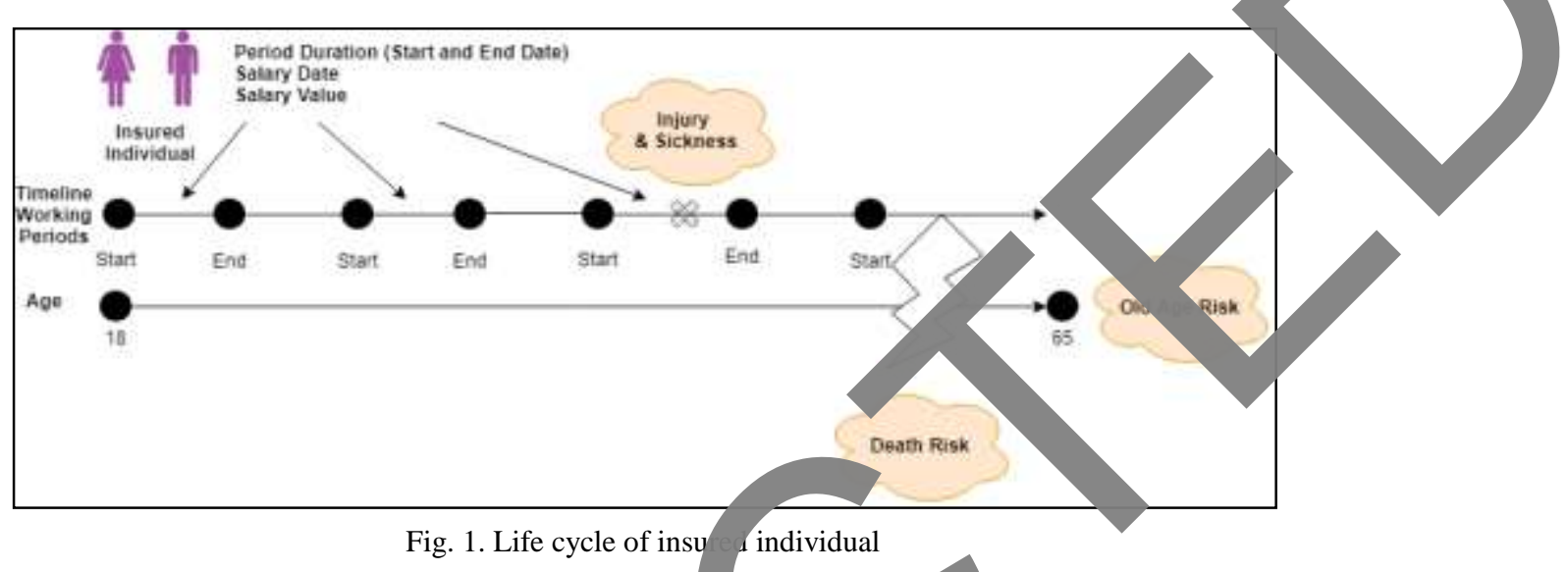

Big Data is a term used to describe a colle on of data that massive in size that grows exponentially over time like inforn ion from so al insurance. The characteristics of Big Data usually include five Y's: Volv c, Velocity, Variety, Veracity, and Value [2].

- Volume. Many data sets a too ran to store or analyse using traditional database technologies and are bein dder or u continuously as well. The National Organization fo cocial Insu (NOSI) in Egypt has big data volumes, which contain the ful ata or sured o on-insured people that are registered in the social insurance sc me. We termined the data from their current datasets and it counted about 46,3887 mends. We found the size of approximately about 3.7 Terabyte

- V city. Whi ata volumes are increasing, data creation and usage speeds are als easing. NOS old systems are not effective to do fast analysis in real time ata to ake decisions $e$ will discuss this point later in the implementation areas in the fra work presentation.

- Variety. m data to website logs, from tweets to visual data such as photos and videos, data con $s$ in numerous shapes and forms. The nature of NOSI's data type oes not vary. NOSI's data is mainly text data, numbers (integers/decimals) a' dates. But in the future after developing NOSI's information systems it may ontain pic es, scanned documents beside these data types.

- racity. Veracity is about ensuring that the insights derived from data are re and valid. NOSI's data contain rubbish data because of the bugs of the data entry applications since a long time ago.

- Value. Data does not have a fundamental value at the simplest level. Only when extracted the insight needed to solve a particular problem or meet a specific need, they become useful. NOSI's data are complex so they needs a lot of development to extract a meaningful value from them. 
After discussing the $5 \mathrm{~V}$ 's of Big Data, we may assign a score from 0 to 5 to determine if the social insurance data is considered as a big data or not. Table 1 shows each $\mathrm{V}$ and its score in NOSI.

Table 1. Big data Vs and its score in NOSI's Data

\begin{tabular}{|c|c|c|}
\hline Big Data Vs & Factor & Score (0-5) \\
\hline Volume & $>1 \mathrm{~TB}$ & 5 \\
\hline Variety & Not Vary & 0 \\
\hline Velocity & Needs Development & 4 \\
\hline Veracity & Needs Development & 3 \\
\hline Value & Needs Development & 1 \\
\hline
\end{tabular}

It is shown in the table above that the final score is 13 poi which is th than half of the total score. So, we considered the social insuran as big data.

This paper proposes a novel big data framework fo gyptic Social insuran. NOSI's data contains data, which need some pre-processing method fter extraction like replacing missing values, standardization and roxtreme da The paper also presents using some mining methods $\mathrm{s}, \mathrm{A}$ as clustering and chs sification algorithms on the Egyptian social insurance d: set.

The rest of the paper organized as follow: Section 2 prese s a literature review of big data mining and big data analytics. Sect 3 presents th ocial insurance big data framework, implements some parts of the fi ework an explains the rest with some insurance use cases. In Secti syptian social insurance dataset and experiment results. Fina , tha Cection 5 presents the conclusion and future work.

\section{Literature rev}

In the following $\mathrm{s}$ ion $\mathrm{y}$ some of the researches related to big data mining and alytics surance.

$\mathrm{Ki}$ and $\mathrm{Cho}$. presented a data governance framework for big data imple $n_{\text {t }}$ on with the n nal pension system. This research carried out a case anay,sis of S Korea's Nâronal Pension Service (NPS). They focused on public tor big data ices to enhance people's quality of life. The procedures of NPS Big Data services consist of four steps: extracting, transforming, cleaning, and loadin A data flow assessment scheme is built and used to handle information flow in ructured form that can be traced via a schematic diagram. The study presented

ar theoret 1 steps of collecting data. However a detailed implementation of these teps is no xplained. The four procedures used in the author's paper will use some - hes eps such as extracting, cleaning and loading in our proposed framework by usi. Social Insurance Big Data (SIBD) in Egypt.

Huss a in and Prie to [4] present a research about big data in the finance and misurance sectors. The research discusses the benefits of analysis of industrial needs in the finance and insurance sectors, benefits like enhancing the levels of customer insight, engagement, and experience. The authors illustrate the available data resources - structured data: transaction data, data on account holdings and 
movements, market data from external providers, securities reference data, price information, and technical indicators; unstructured data - daily stock, feeds, company announcements, online news media, articles, and customers' feedback. The most important point the authors focus on are technical requirements like data extraction quality, acquisition, integration/sharing, privacy, and security. Overall, the resear presents a good background in the insurance and finance sectors. The resarch presents a good methodology to use all insurance customer data; struct $d$ and unstructured to build a good vision to enhance their services. In our fra work, we will use the structured data such as id, periods, and pensions of the ins dindivid But the unstructured data is not applicable in the social insurance sys in oypt because of the lack of resources and technologies.

Song and Ryu [5] present a big data analysis framer $k$ for healthc. and social sectors separately and assign them tentative names: " alt " sk analysis ce. and integrated social welfare service. The authors face some obs les in applying their framework. First, government ministries and agencies managen t committee is needed to correctly manage big data for healt' ate and welfare serv because big data need to be managed in an integrated $\mathrm{w}$. Second, a cooperative system with private organizations must be established at maintains structured big data associated with healthcare and welfare service Most big data lated to healthcare and welfare services are owned solely by the blic sector. lird, technology for analysing and processing large info ation on heà re an velfare facilities needs to be developed. The study shows e on thes thave occurred in related fields similar to social insurance such as $\mathrm{h}$ thcare elfare. The study gives a starting point to the proposed framework to e an we Egyptian social insurance scheme.

Tsai et al. [6] pre ig data ey. The researchers analyse data analytics studies from the con ational ta analy to the new big data analysis. Three aspects of their framew are sur harized: i ut, evaluation, and output. The paper concentrates on form and results-oriented problems from the viewpoint o de big nalytics system and platform. Also, research offers a brief introduct ${ }^{t}$ to the big a mining algorithms consisting of clustering, classification, and $r$ attern minin ochnologies from the perspective of the data mining pro $-m$. The search presen. a good mixture between big data analytics and mining hich can be us as a good reference in the proposed framework. The framework will contain some cases that can be applied in the social insurance data such as classif cation, clusterng, statistical and actuarial analysis.

in o o 1 a et al. [7] present big data challenges and possibilities using case studies were im emented in the South African insurance industry and the technology nd instry nts required to analyse Big Data. They also discuss the roles that
and int ction to data governance and laws as well as a possible perspective on what the future might hold is presented. The research reflects full information about big ata insurance and supports this paper in the organization of our framework. The big data use cases in insurance will be presented in the framework like customer segmentation, risk assessment, and loss reduction. 
Yenkar and Bartere [8] published a review on data mining with big data. The publishers implement heterogeneous combination learning in the big data revolution and also the data-driven model of data mining involves extracting and analysing large quantities of data to create large data models. The techniques come from artificial intelligence grounds and stats with a bit of database managemen Normally, the data-mining goal is either to predict or classify. The idea is to or anize data into sets in classification. The plan is to predict a continuous variable's $f_{4}$ uency in prediction. The research supports us to build our framework in socir nsurance after the data extraction and integration the framework is divided int wo branc' - big data mining which contains classification and big data analytirs, w h co ans actuarial analytics that aims to predict the expenditure of pensio in the

\section{SIBD framework}

The proposed Social Insurance Big Data ( $\mathrm{S}^{\prime}$ ) Hramework comp social insurance big data extraction and collecting of ases to be used in social insurance and how to apply it in Egypt. The framewor as shown in F 2 aims to enhance social insurance services, help the insurers an ctuaries to tal a good decision in the fast and right way and give us more insight to the soci data. The first stage includes the data extraction and co tion steps us. The cond stage contains the data integration and selection of the xtra data and creating the dataset. The third stage is pre-processing of the datas The tonsists of big data mining and analytics. The big data minin se on is arvided into classification and clustering. Big data an onsist o atistical and actuarial analysis and lifetime value prediction.

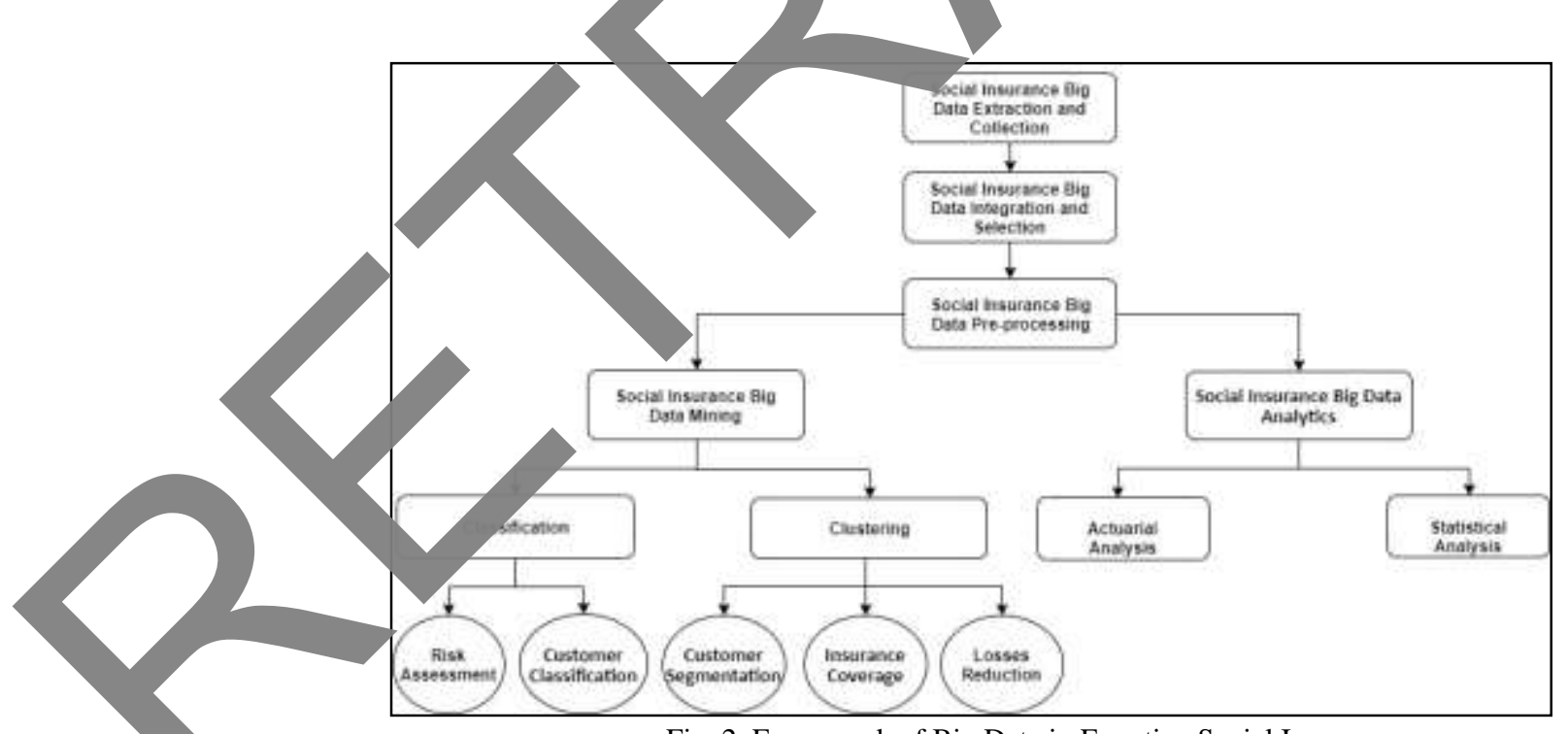

Fig. 2. Framework of Big Data in Egyptian Social Insurance 


\subsection{SIBD extraction and collection}

National Organization for Social Insurance (NOSI) is the only authority responsible for Egyptian Social Insurance data. The architecture of a NOSI information system mainly consists of a centralized IBM mainframe. The type of database that stores $\mathrm{d}$ ? on it is hierarchal. Data extraction and collection from mainframe is going through the following stages:

- Choosing the hierarchical mainframe schema that will be extract data files.

- Creating batch programs that are responsible to read the date ad write

- Initiating batch processing which compilation of the $b$ ch pro from production control to computer operation.

- Provide the necessary spaces, which presented by ane tapes. D. written in tape blocks.

- Data is integrated from tapes to the sequential dataset.

- Transfer data from sequential dataset to $P$ su er as flat text

\subsection{SIBD integration and selection}

The integration and selection of insurance dat ontain seve important steps to produce the final dataset that will used in clus an ansification. The first step is to create the same database bro he hierarcmicar mainframe database with the same attribute names. The secon step is integration services to insert data from text files into database tables. Th thir step is wing some SQL queries to select important columns fron ase tab and finally - collecting all data in NOSI dataset (Fig. 3).

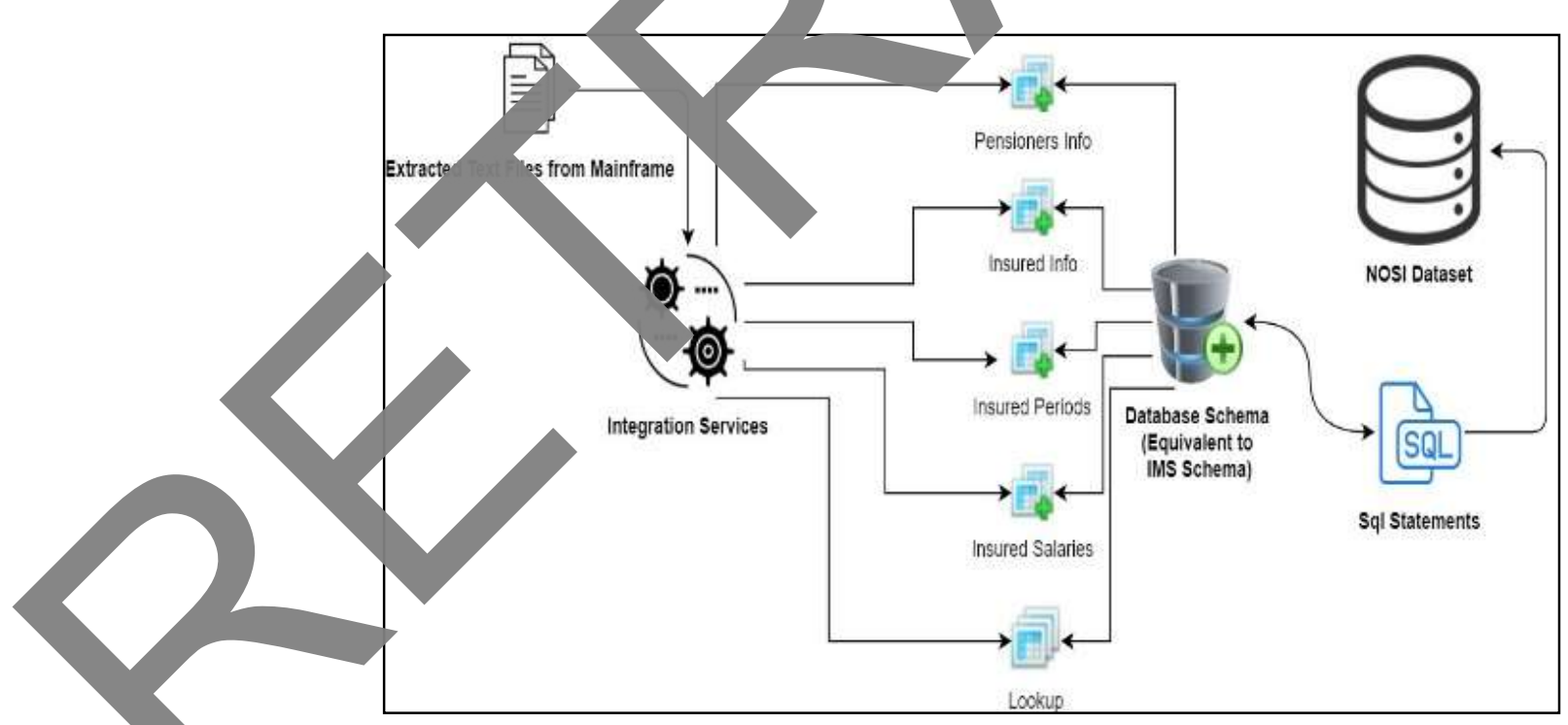

Fig. 3. Data integration and selection steps 
NOSI's data consist of four basic tables: Insured Individuals Info, their Insured Periods, and Salaries. Also, Pensioners Individuals Info with some lookup tables like Cities, Job Categories, and Sectors. In the integration and selection processes, Microsoft SQL Server Management Studio is used to create database tables and NOS dataset. Also, Microsoft SQL Server Integration Services is helpful in the insertio of the bulk of data in a short processing time. Every integration container con ${ }^{\prime}$ ts of the File System Task, which is responsible for integrating the text file from ae FTP server location to the database tables. Data Flow Task, which is used f handlin every database table and datatypes for each table to be inserted succe dlly.

Table 2 shows sample data selected from the integrated data, whic ont the basic attributes that represent the life cycle of the insured indiy" aal. Not e "Job End Date" column is empty which means that the insured in dual is still h ying and does not have a job end date.

\begin{tabular}{|c|c|c|c|c|c|}
\hline Gender & Age & Sector & Total_Period & Job_Stam Date & Job_End_ \\
\hline Male & 64 & Private & 21.3 & $1 \quad-0<-03$ & $2010-05-2$ \\
\hline Male & 40 & Private & 16.5 & $015-09-01$ & \\
\hline Female & 61 & Public & 11.1 & 2002-01-01 & $12-07-31$ \\
\hline Male & 35 & Private & 10 & $2017-08-17$ & \\
\hline Male & 77 & Private & 31.1 & 976-01-30 & $07-03-06$ \\
\hline Male & 53 & Private & 12.2 & 2. $-02-08$ & \\
\hline Male & 68 & Private & & 200 & 2011-09-11 \\
\hline
\end{tabular}

\subsection{SIBD pre-processing}

There are some pre-proce tasks to and develop the accuracy of the data mining algorithms. $\mathrm{T} r$ are ne basi asks in big data pre-processing such as data cleaning, complete se missin values, a data normalization [9]. Data cleaning is the biggest chall $e$ in SIP find errors in lata conversion ecause some rows have illogical data. The unknown americ $\mathrm{h}$ ing values will be replaced by mean values. Data normal $\quad$ in is used to $\mathrm{h}$ dle characteristics on a different scale; otherwise, it may redy the ciency of an sally significant attribute due to other characteristics having values bigger scale. This will be illustrated in the experiment later.

\subsection{SIBD mining}

$\mathrm{Cly}$ ring and classification are the most frequently used techniques in big data ang. The techniques were chosen based on the description of the issue and our nterest in xperimenting with two separate methodologies, whose fundamental yre summarized next.

\subsubsection{Classification}

Classification is a binary modelling method that includes dividing the set of data into precisely two subgroups (or "nodes") that are more homogeneous to the reaction variable than the original set of information. It is recursive because for each of the resulting nodes the process is repeated. The data divided into two nodes, are then 
compared to all feasible splits for all values for all factors included in the assessment and an exhaustive search is conducted through them all, choosing the split that separates information into two nodes with the greatest degree of homogeneity. There are some of case studies in Egyptian social insurance using the classificatior techniques such as risk assessment and customer classification. They are explain below.

\subsubsection{Risk assessment}

In general, risk assessment is the process of estimating and evaluat the ris in addition, it detects the possibility of the occurrence of some ng b $^{\prime}$ al or harmful to the individual at a certain time. There are two typ of risk ass ment: Risk-Taking Model which is focused on normal things ights, choice nd participation of the individual and Risk Minimization M el fo d on danger a health. Social insurance is typically considered as the second type or $k$ assessment. We can divide the insured individuals in Egyn three categ s: Insured individuals from 18 to 24 years, from 25 to 45 ad, from 46 to 65 . Eve. category has its social risk assessment from death, retir nent, and disability. For example, in the first category the death, retirement, and sability rates 11 be lower. In the second category, the retirement will be lower th death and dis ility because in this category there are some dangerous jobs such a ilitary, $\mathrm{dr}$ ing, and metallurgic jobs. In the last category, the retir ate is highu ath disability. The classification in risk assessment ca suppo in the estimation of expenditure of pensions of the insured individuals.

\subsubsection{Customer cla}

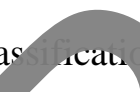

The classification gorithm's ask is to d out how that set of characteristics can lead us to take a tain de trovichals in Egyptian Social Insurance can be classified by ing su the dataset attributes such as insurance number, age, total periods. $F$ example, he analyst wants to predict the number of pensioners that have a retirement a there is a rule of early retirement, which is the insured indi aual, have at leas, 20 years or more of total working periods as shown in Table 3.

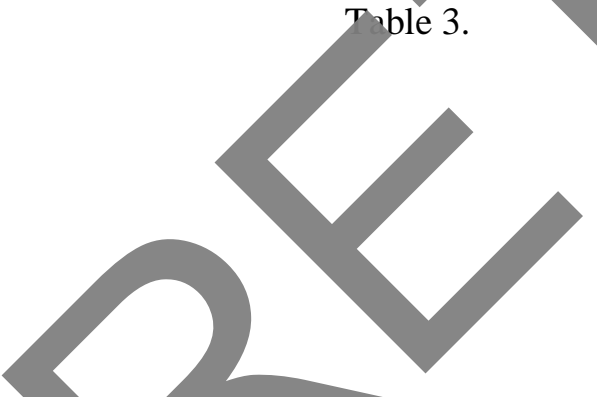

Table 3. Selected data for customer classification

\begin{tabular}{|c|c|c|c|}
\hline ender & Age & Total_period & Deserve pension \\
\hline 1 & 64 & 21.3 & YES \\
\hline 1 & 40 & 8.5 & NO \\
\hline 2 & 61 & 11.1 & NO \\
\hline 1 & 35 & 15 & NO \\
\hline 1 & 77 & 31.1 & YES \\
\hline 1 & 53 & 12.2 & NO \\
\hline 1 & 68 & 40.1 & YES \\
\hline
\end{tabular}

The Gender, Age, and Total Period predictor columns determine the value of the Deserve Pension (predictor attribute). The predictor attribute is recognized in a training set. The classification algorithm then attempts to determine how the predictor attribute value was achieved. 


\subsubsection{Clustering}

Clustering is a process of dividing similar data into objects called clusters. There are many types of clustering algorithms in big data mining such as partitioning, hierarchical, density, grid model, and constraint based clustering algorithms [10]. $\mathrm{T}^{\mathrm{l}}$ use cases in social insurance using clustering techniques are customer segmentation, insurance coverage and losses reduction.

\subsubsection{Customer segmentation}

To offer the insurer the opportunity to obtain understanding about the o ome om various perspectives, we can generate one or more dimensions sy as dem phics, behaviour, and value. In each dimension, the clustering methe nust follow th ame schema. The variables used for segmentation should be os based on a s criteria [11]. The demographic segmentation in Egyp an So Insurance can consists of young, adult and old men and women. The segmentatio of behaviour

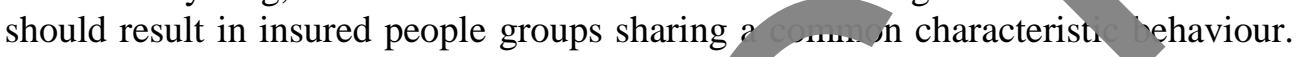
This behaviour should identify insured individ as that can be managed in the same manner, but this should be managed dif rently from ther segments. The segmentation's third and last dimension is ue. This dime sion concludes the insured is going to be financially compensated en risks occ

\subsubsection{Insurance coverage}

The amount to which risks are cove for individual is called insurance coverage. Insurance covernoe represe s level or effectiveness of implementing social insurance polic nea ed by th percentage of the insured population to the total working-age pulation nder so 1 insurance policies [12]. The working population in Eg can be ided into ar clusters. The first cluster is about the insured youtb $\&$ aa wi tow second one is about insured employees with high ome. Th ard one collects old insured employees before retirement. The fou cluster can bo out the pensioners.

\subsubsection{Losso duction}

is mentioned bet the idea of a social insurance scheme is to collect a contribution from insured indivia als and benefit them if any risks happened to them. Clustering met', like partitioning by using K-means algorithm, can estimate the expenditure of gyptian pensions by calculation of the pension rates, and what would happen if rese rates increased or decreased. The pension rates can affect the expenditure

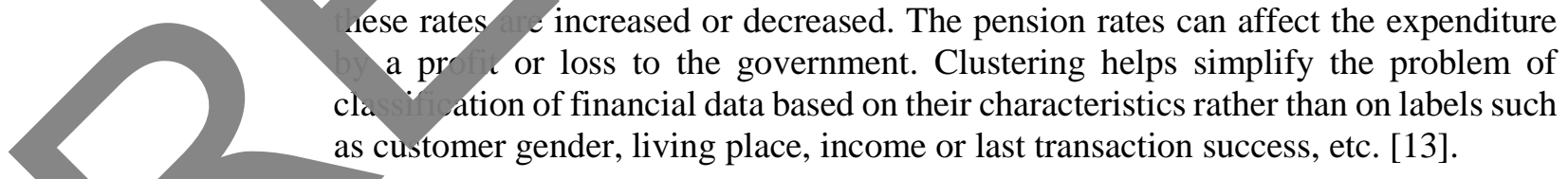

\subsection{SIBD analytics}

Big data analytics mainly encompasses big data analytical methods, systematic big data architecture, and analytical software. Data analysis in big data is the most 
important step to explore meaningful values, make suggestions and make decisions. Analysis of data can explore potential values. However, data analysis is a wide, dynamic and very complex area [14]. Social insurance with big data analytics will be useful in some type of analysis like statistical analysis, and actuarial analysis.

\subsubsection{Statistical analysis}

The statistical analysis for big data can be grouped into two main types: $r$ ampling and divide-conquer. Resampling is a straightforward method created serve ty fundamentals. First, resampling offers a deviation from the fix hypoth is underlying many statistical processes. Second, resampling off a s or estimating the distribution of statistics that are very complicat Therefore, cause of the second fundamental, it will be useful to use res ing to estima the distribution of pensions over the insured individuals. Tb echn of dividing a $A$ conquering usually has three steps: (1) partitioning a large data set $K$ blocks; (2) processing each block individually (potentially in llol), and (3) as egating the alternatives from each block to create a final s ation to the complete -ormation [15]. A divide and conquer strategy can cause ficiency and enhancement of social insurance.

Actuarial models aim at demographic a financial pr ections of pension systems that were generally derived from hels that d been applied to occupational pension schemes co oroups of oased on demographic variables, economic variables, a soc hehavioural) variables of workers. Actuaries need to recognize that de lopin an ate and definitive formula of human behaviour is complinated and ways feasible. Accordingly, in relation to predictive analytics, ar arla thods i d to considerably improve their knowledge of expected condu or occul nces an upport their policies and decisions [16]. Therefore, big d analytic sill cuppor actuaries in their work. One of the most important go for for ar is impung a year-by-year simulation technique to predict fut expense 1]. For instance, we can use, the following general equation that is $v$ in their simul an technique to predict the future expenditure of pensions in $\mathrm{E}$

$$
\text { Next Year Expenditure }=\text { Current Year's Expenditure } \times \text { Survival rate } \times
$$

$\times$ Adjustment 1 or + Cost of New Pensions Projected for Award Next Year.

T base of contributions is calculated by multiplying the assumed amount of ac insured individuals by the projected average insurable income and the ntribution te of the system (contribution factor):

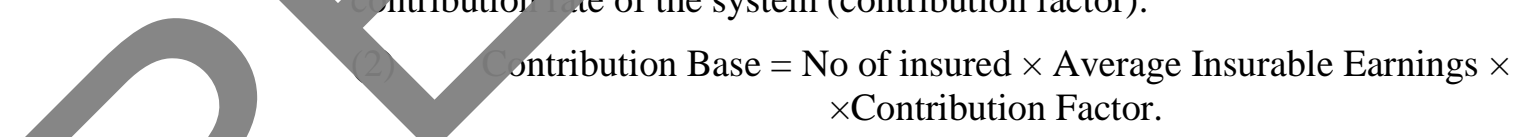
$\times$ Contribution Factor.

At this point, the research discusses the areas of the proposed framework. Next section presents an experiment with the implementation of some parts in the framework. 


\section{Experiment}

The experiment section contains a description of the Egyptian Social Insurance dataset and its features. The pre-processing methods that can be applied to the datase such as imputation, standardization, and outlier, and clustering between data node. Finally, applying a classification algorithm and measure accuracy, $f$-measure call, and precision. In the experiment, we used two miner tools WEKA and Or

\subsection{Dataset}

Due to the hugeness of Egyptian Social Insurance datasets, th experimo tataset was carefully constructed based on the fundamentals of socir surance and it asic features. Also, we decided to get the data of the individu fro 90 years only, the whole data. In short, the dataset includes the insured ndividua tho have actual work periods and the target feature determines whethor they are ben iaries of the insurance system or not? In other words, are th caking pensions or hable 4 describes the main characteristics of the datase

Table 4. Dataset characteristics

\begin{tabular}{|c|c|}
\hline Dataset Characteristics & Multivariate \\
\hline Attribute Characteristics & Numeri aminal an ate \\
\hline Associated Tasks & \\
\hline Number of Instances & \\
\hline Number of features & \\
\hline Extraction Date & $201,-11-03$ \\
\hline
\end{tabular}

\subsection{Dataset features cscripti}

Dataset features ttributes onmecent the basic information about the insured individual su as her se, genacr, $\mathrm{s} / \mathrm{her}$ sector of work, etc.

Table slustrates description of each dataset features and the target.

Table 5 at. features

$\mathrm{Fe}_{\mathrm{a}}$ Description

ge

Gender $\quad$ is ender Male or Female

\begin{tabular}{|l|l}
\hline City & It co \\
\hline
\end{tabular}

\begin{tabular}{|l|l}
\hline Sect & The insured individual work sector such as public, private, etc.
\end{tabular}

$\mathrm{J}$ ategory The category of work of the insured individual like Doctors, Engineers,

Carpenters, etc.

Last Job St $\quad$ The last starting date of his/her work

st Jor ad The last ending date of his/her work. As mentioned before, the job end date may contain empty values and this means that the insured individual is still working

Fuli nsurance Calculated by subtracting insured working duration dates the start date and end Deriods $\quad$ date and sum the result durations

Target Description

\begin{tabular}{l|l}
\hline Takes A & It contains YES or NO values. YES means the insured individual takes a
\end{tabular}

Pension $\quad$ pension, NO means the insured not taking a pension 


\subsection{Dataset pre-processing}

Before applying the pre-processing methods clarification of feature statistics is needed to decide which pre-processing method is suitable for the dataset. Feature statistics consists of centre (average), dispersion (median), minimum, maximum, a the missing values percentage. The following Table 6 expounds the dataset featuro statistics.

Table 6. Feature statistics

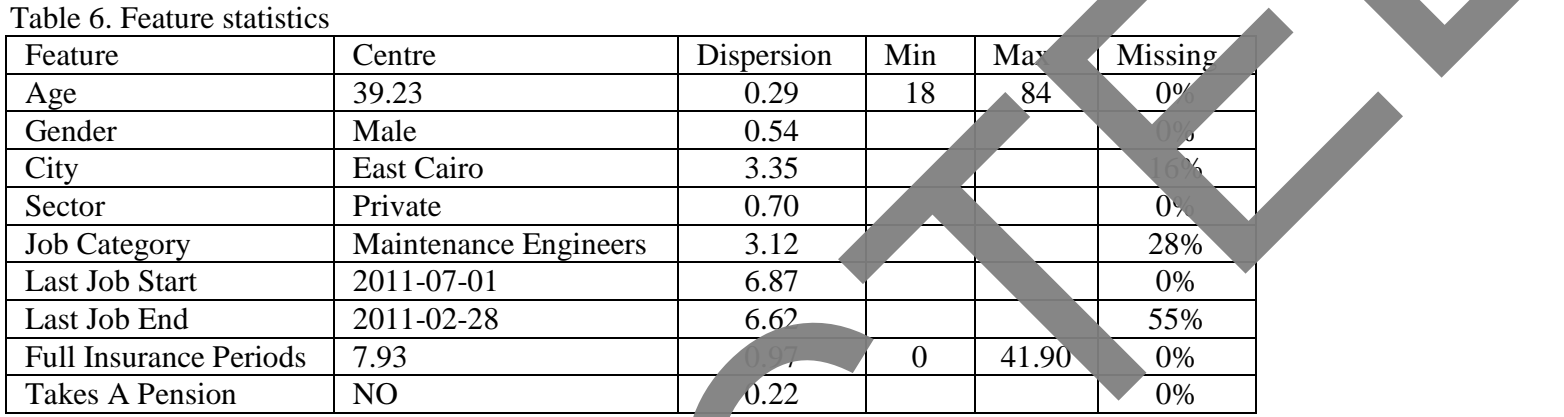

4.3.1. Replacing missing data with mean impu tion

This replaces the missing value with the mean or erage samp or model depending on the data being distributed $[1 \%$ The feature the ore than $50 \%$ will be removed from the dataset because hen sing values are large in number, all of these values will be replaced by same value, that is mean, and contributes to a shift in the distributic fo 1. Thererore, the "Last Job End" feature will be removed. By .... VEKA place missing values" filter, all missing percentages is turne $00 \%$.

\subsubsection{Z-score-non izatir}

This met also ca "Standardization". The method aims at rescaling the charact ics of data be en zero and one. If $A$ represents the mean of the values of $\mathrm{f}$ are nd $\sigma_{A}$ is the andard deviation, original value $v$ of $A$, then $A$ is normalized to sing the following equation:

$$
V^{\prime}=\frac{\mathrm{v}-\overline{\mathrm{A}}}{\sigma_{A}} .
$$

applying this standardization on the feature values, present a mean equal to and a standard deviation of one [19]. After using the "Standardization" filer in EKA, the ature "Age" is ranged from -3.342 to 7.09. Also, the "Full Insurance eriod" f are is ranged from -1.017 to 4.48 .

4.3. Outlier/ extreme values

1 outlier is an occurrence in dataset values that have distance from other values. Extreme values are either too large or too small values [20]. In the dataset were found 19676 instances considered as outlier values and there are no extreme values. The instances were removed by "remove with value" filer. So, the number of instances reduced from $13,800,427$ to $13,780,751$. 


\subsection{Clustering}

In clustering on the Egyptian social insurance dataset, we decided to use the K-means clustering. K-means is a method by which observations are grouped into a specif number of disjoint clusters. K refers to the specified number of clusters. There aro different distance measures that are used to determine which observation $t o$ be appended to which cluster.

To detect the number of suitable clusters on the dataset the K-me calculat the silhouette score of each cluster. The silhouette value is a similarity asure an object is to its own cluster compared to other clusters.

In the next formula, we can explain a(i) as a measure ond how fully assigned to its cluster; $b(i)$ is the minimum average distanc een $b(i)$ and data point in other clusters that are not included in $\mathrm{K}[21$

$$
s(i)=\frac{b(i)-a(i)}{\max \{a(i), b(i)\}} \text { if }\left|C_{j}\right|>1 .
$$

The silhouette score range from -1 to +1 . Tb igh value indicates the he object fits well with its own cluster and is negatively igned with neighbouring clusters. If most objects have a high value, then the con suration for cl ering is suitable. If many points have a low or negative value, th there may be o many or too few clusters in the cluster configuration. The $\mathrm{K}-\mathrm{h}$ ns implen itation presents the following results that are explaine Table 7 whic the silhouette scores of two or more clusters on the dataset.

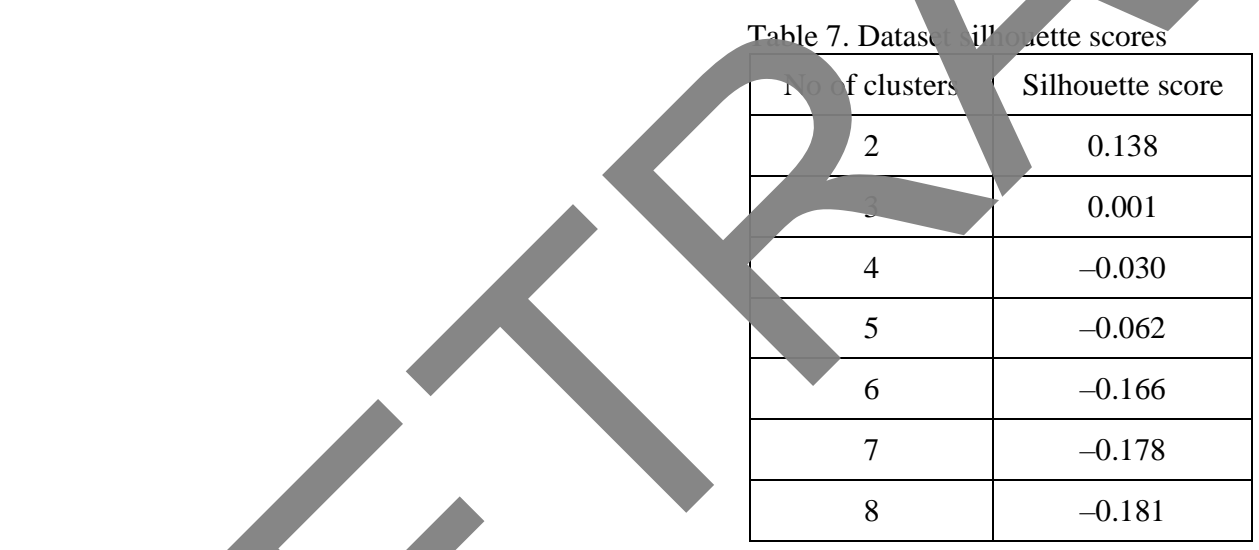

From th able above we found that two clusters would be suitable for the dataset cause cy have a higher score from other numbers of clusters. In cluster vi vion of the K-means algorithm results, we used the scatter plot to describe the iformative projection between clusters. The score plots detected three cormative projections. The first projection is between "Age" and "Full Insured Period" features; the second projection - between "Age" and "Silhouette"; the third one - between "Full Insured Period" and "Silhouette". Fig. 4 contains scatter plot of the three projections respectively. 


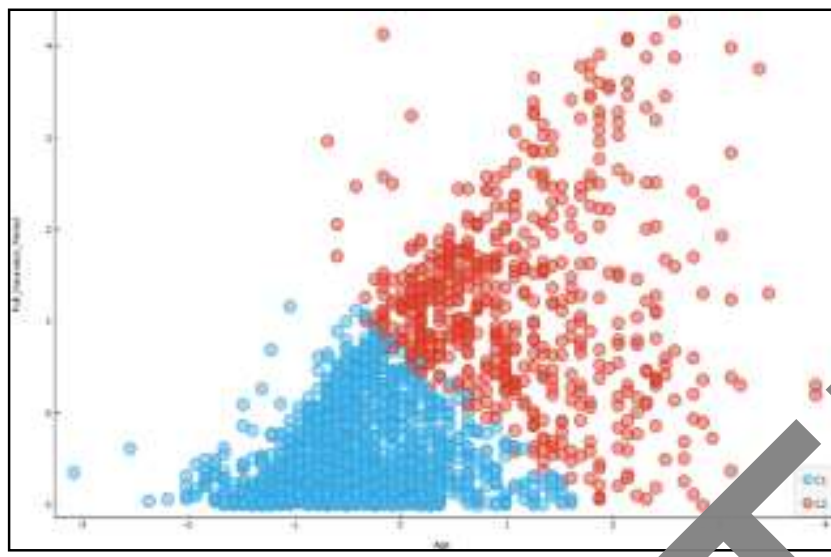

(a)
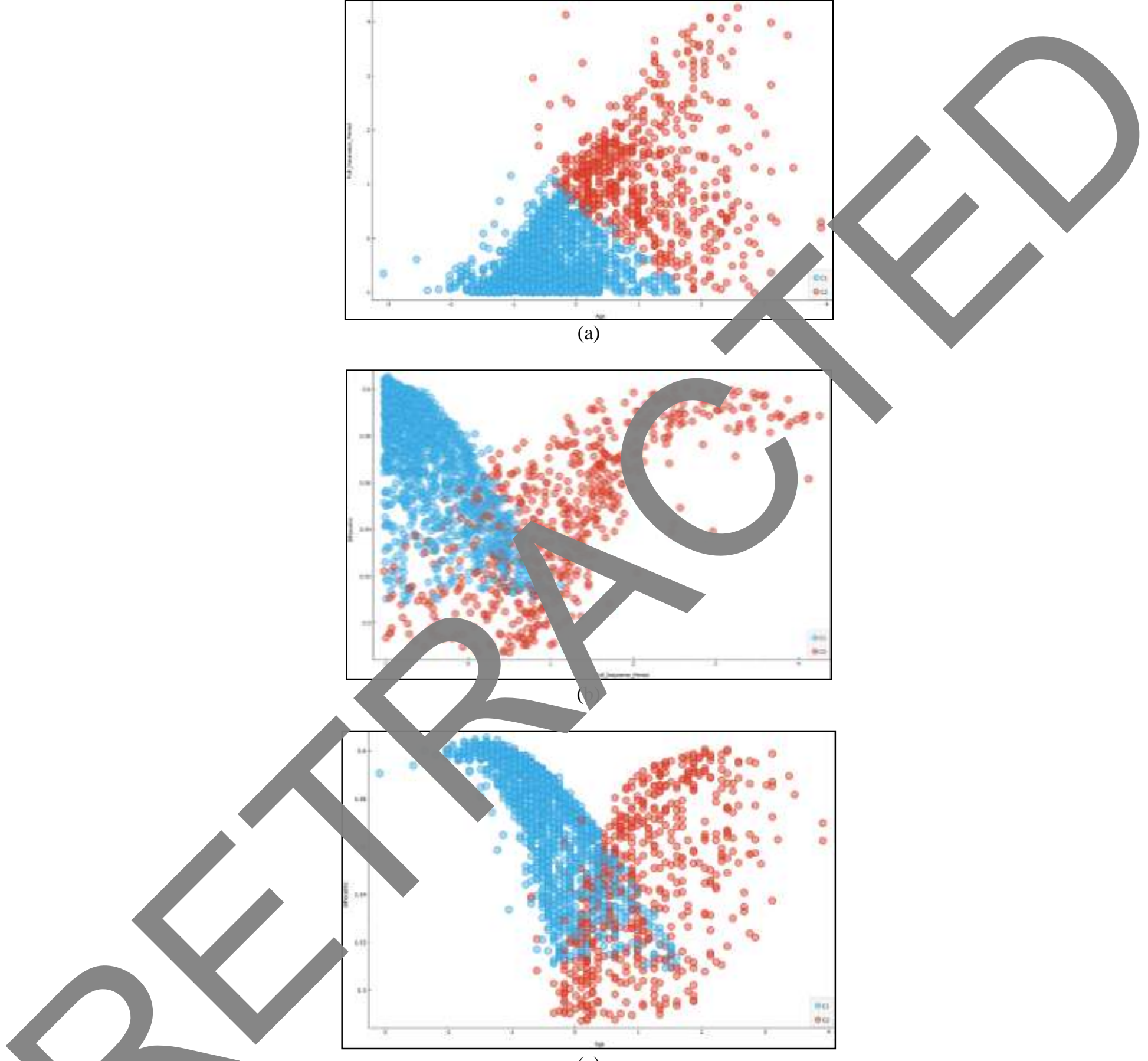

(c)

Fig. 4. Scatter plot of age and full insured period (a); Scatter plot of full insured period and silhouette (b); Scatter plot of age and silhouette (c) 


\subsection{Classification}

Data classification consists of two main stages. The first stage is to create a classification model that involves the learning process, pick the algorithm to construct a classification model, and use the training set to construct a classification mod The second stage is to use the classification system, which involves the classification method analysis and the classification model that can be applied to the new data if the reliability is appropriate. The dataset is divided into a training set an test set; $80 \%$ of the data has been used for training, and $20 \%$ of the data has $n$ used $f$ testing.

The Support Vector Machine (SVM) is the chosen algorithm the c. ation experiment. The SVM divides all data objects into two catego s in a featu space. The data object in SVM algorithm is defined, the features $\left\{x_{1}, \ldots, x_{i}\right\}$ and a label as $y_{i}$. SVM treats every data object as a point in the ace o, features so t. $y$ the object belongs to any class. So, when the class label is $y_{i}=1$ tho he data object belongs to the class, or when $y_{i}=-1$ then the data oes not belon. o the class. Therefore the general formula for the data [22]

$$
\left\{\left(x_{i}, y_{i}\right) \mid x_{i} \in \mathfrak{R}^{p}, y_{i} \in(-1,+1)\right\}_{i=1}^{n}
$$

After applying the SVM algorithm on the taset, the accui $y$, precision, recall, and $f$-measure will be the evaluation measuren ts of the clas ication experiment, and accuracy, which is the ratio of correct predict Precisi is the ratio of correct positive predictions. The recall is the of posinabelled instances, also predicted as positive. Finally, $f$-n asure hines precision and recall in the harmonic mean of precision and red $1 . T 2<s$ s the classification measures result of the dataset.

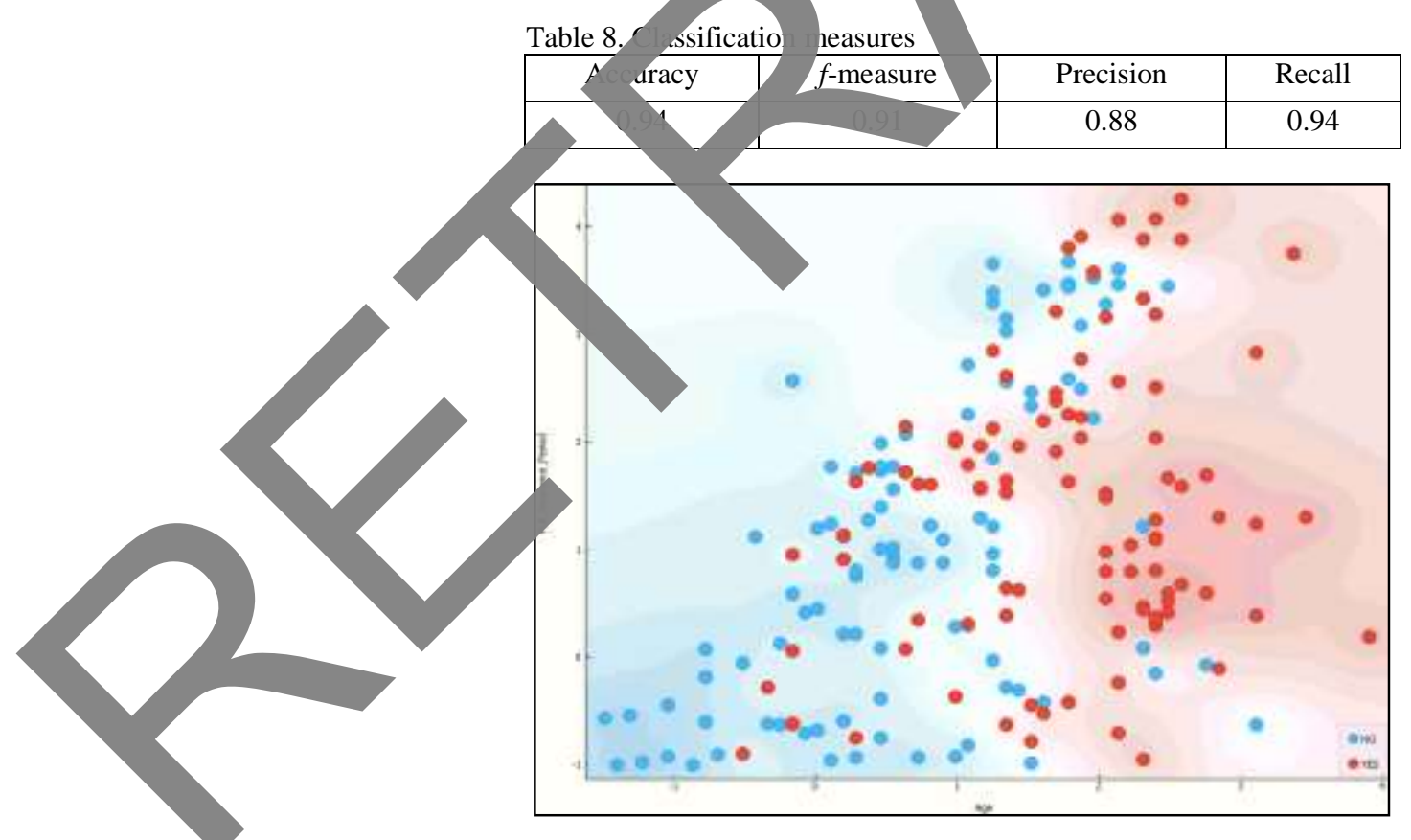

Fig. 5. Scatter plot for SVM Algorithm 


\section{Conclusion and future work}

This paper proposes a new framework for big data in Egyptian Social insurance to collect all the basic steps and methods for better benefits to the insured people. $\mathrm{Tb}$ paper presents a literature review of some researches about big data mining and bi data analytics in social insurance. The paper implements some parts of the pre nted framework through an experiment and explains the rest of it. Finally, the esearch overall tries to spotlight on social insurance field and give a mixture of $y, g$ big dat mining and analytics to help the insurers and the actuaries to get the b decision the insured individuals.

For future work, we will extend this work with more pre-p essing n ods on the selected social insurance dataset. Furthermore, we will y ome big data ing techniques by focusing on classification algorithms and ar y so of the superv learning algorithms, such as decision tree, $\mathrm{CN} 2$ rule induction, d naïve Bayes algorithms, and compare measurement between them

Also, the challenges that faces the researche an bo solved by the marizing following points and this can consider as our $\mathrm{f}$ are framework updates:

- Structuring of big data sampling meth is is important reduce the amount of big data to a manageable size.

- A higher level of data science expertis is needed implement big data strategies to determine how long ata need to as some data are useful in making long-term decisions, wh oun are not applicable. Therefore, the importance of data selection in our $\mathrm{t}$ newo experience in business rules of social insurance.

- Data mining a need de to be loaded into the main memory even if having super-large in mem to stor 11 data for computing. So, the development of data collection d integr on is impor at for big data mining in the framework.

- The i^por e o cealms wledge indexing framework to ensure realtime data $r$ sitoring c classification for big data applications.

U.S. Census Burea USCB]. Social Insurance and Human Services. Section 11. 2004, pp. 355-381.

2. Gantz, J., D. Re se l. Extracting Value from Chaos, in IDC's Digital Universe Study. Sponsored by EMC, 2011.

3. m, H. Y., J. C h o. Data Governance Framework for Big Data Implementation with NPS Case Analy in Korea. - Journal of Business and Retail Management Research, Vol. 12, 2018, No

H u s n, K., E. Pri e t o. Big Data in the Finance and Insurance Sectors. Book of New Horizons or a Data-Driven Economy, 2016, pp. 209-223.

5. S g, T., S. R y u. Big Data Analysis Framework for Healthcare and Social Sectors in Korea. Healthcare Informatics Research, Vol. 21, 2015, No 1, pp. 3-9.

T s a i, C. W., C. F. L a i, H. C. C h a o, A. V. V a s i l a k o s. Big Data Analytics: A Survey. - Journal of Big Data, Vol. 2, 2015, No 21, pp. 1-32.

7. B hoola, K., T. Madzhadzhi, J. Narayan, S. Strydom, H. He erden. Insurance Regulation in Africa: Impact on Insurance and Growth Strategies. Actuarial Society of South Africa's, Cape Town International Convention Centre, 2014, pp.145-196. 
8. Y e n k a r, V., M. B a r t e re. Review on Data Mining with Big Data. - International Journal of Computer Science and Mobile Computing, Vol. 3, 2014, Issue 4, pp. 97-102.

9. García, S., S. R a mír e z-Galle g o, J. Lu eng o, J. M. B enít e z, F. Her re ra. Big Data Preprocessing: Methods and Prospects. BMC Big Data Analytics, 2016, pp. 1-22.

10. T i r u ve e d hul a, S., C. M. S. R a n i, V. N a r a y a n a. A Survey on Clustering Techniques fr Big Data Mining. - Indian Journal of Science and Technology, Vol 9, 2016, No 3, pp. 1-12.

11. B ü c k e r, T. Customer Clustering in the Insurance Sector by Means of Unsupervised Machine Learning. Internship Report, 2016, pp. 1-112.

12. International Labour Organization [ILO]. Social Insurance: Enhancing Social Secur Right for Everyone. - Policy Brief, Vol. 3, 2014.

13. Ca i, F., N. Le-Kh a c, T. Ke ch adi. Clustering Approaches for Financial a Analys Survey. School of Computer Science \& Informatics, 2016.

14. Chah a l, H., P. Guli a. Big Data Analytics. - Research Journal of Co ater an mation Technology Sciences, Vol. 4, 2016, pp. 1-4.

15. W a n g, C., M. Che n, E. S chif a n o, J. W u, J. Y an. Statistica hods and Compu fo Big Data. - Statistics and Its Interface, 2016, pp. 399-414.

16. American Academy of Actuaries [AAA]. Big Data and the Rou of the A y. Big Data Task Force, 2018.

17. International Labor Office [ILO]. ILO Pension Model Te uide, 2018.

18. J a d h a v, A., D. P r a m od, K. R a m a n a th a n. Co arison of Performance of Da imputation Methods for Numeric Dataset. - International J mal in Applied Artificial Intelligence, 2019, pp. 913-933.

19. García, S., J. Lueng o, F. Herrera. Preproc ing in Data Mini Springer International Publishing, Switzerland, 2015.

20. A g g a rw a 1, C. C. Outlier Analysis. Second Edition. ger, Cham 016.

21. A mori ma, R. C., C. Hennig b. ring the Numb ers in Data Sets with Noise Features Using Feature Rescaling actor formation Sciences Journal, 2016, pp. 1-34.

22. B ridge la 11, R. Introduction to Supp Vector Lecture Notes, 2017, pp. 1-18.

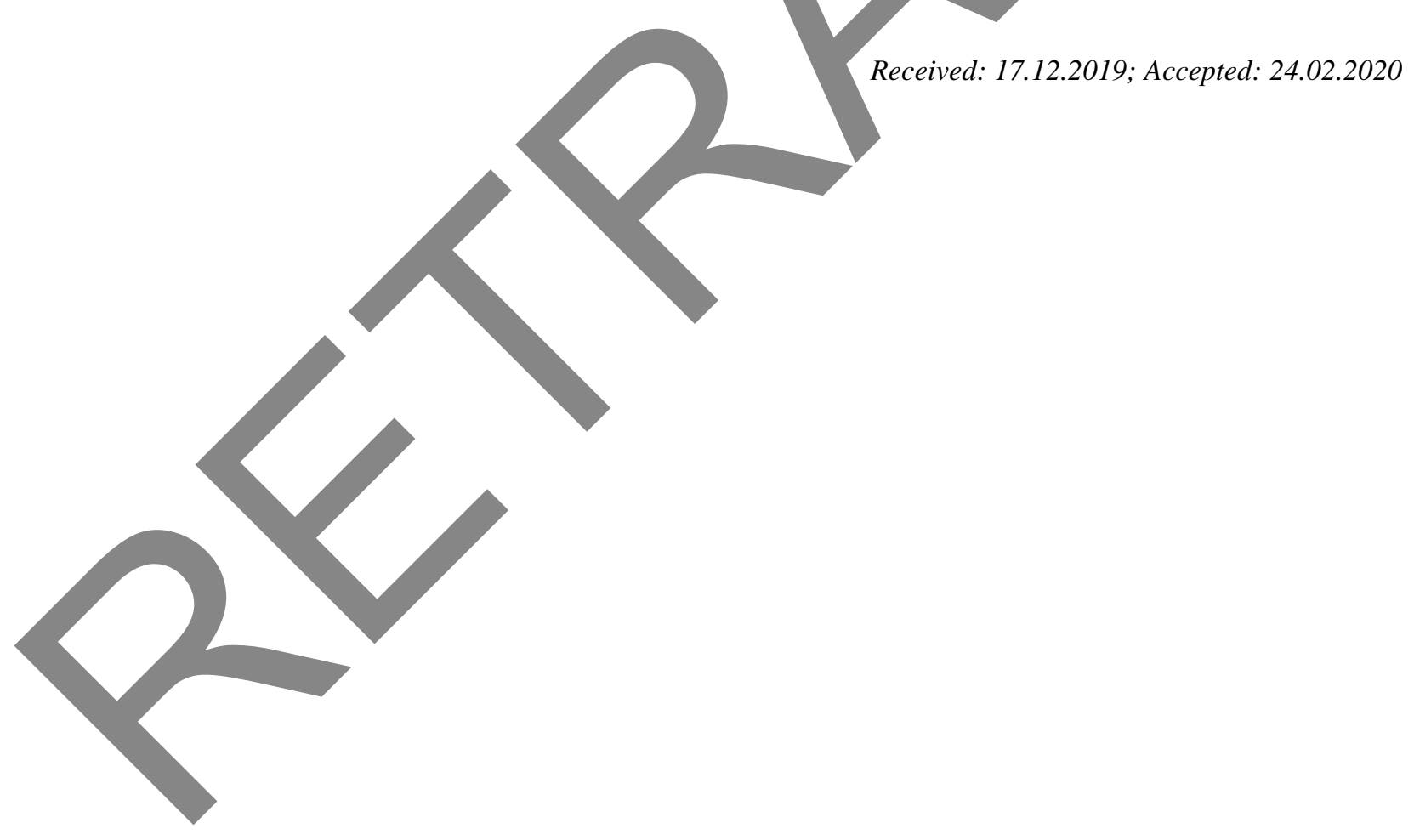

